\title{
First heavy ion beam tests with a superconducting multigap $\mathrm{CH}$ cavity
}

\author{
W. Barth, ${ }^{1,2, *}$ K. Aulenbacher, ${ }^{3}$ M. Basten, ${ }^{4}$ M. Busch, ${ }^{4}$ F. Dziuba, ${ }^{1}$ V. Gettmann, ${ }^{1}$ \\ M. Heilmann, ${ }^{2}$ T. Kürzeder, ${ }^{1}$ M. Miski-Oglu, ${ }^{1}$ H. Podlech, ${ }^{4}$ A. Rubin, ${ }^{2}$ \\ A. Schnase, ${ }^{2}$ M. Schwarz, ${ }^{4}$ and S. Yaramyshev ${ }^{2}$ \\ ${ }^{1}$ Helmholtz Institute Mainz, 55099 Mainz, Germany \\ ${ }^{2}$ GSI Helmholtzzentrum für Schwerionenforschung, 64291 Darmstadt, Germany \\ ${ }^{3}$ Johannes Gutenberg-Universität Mainz, 55099 Mainz, Germany \\ ${ }^{4}$ IAP Goethe-Universität Frankfurt, 60438 Frankfurt, Germany
}

(Received 8 November 2017; published 27 February 2018)

\begin{abstract}
Very compact accelerating-focusing structures, as well as short focusing periods, high accelerating gradients and short drift spaces are strongly required for superconducting (sc) accelerator sections operating at low and medium energies for continuous wave (cw) heavy ion beams. To keep the GSI-super heavy element (SHE) program competitive on a high level and even beyond, a standalone sc $\mathrm{cw}$ linac (Helmholtz linear accelerator) in combination with the GSI high charge state injector (HLI), upgraded for cw operation, is envisaged. Recently the first linac section (financed by Helmholtz Institute Mainz (HIM) and GSI) as a demonstration of the capability of $217 \mathrm{MHz}$ multigap crossbar H-mode structures (CH) has been commissioned and extensively tested with heavy ion beam from the HLI. The demonstrator setup reached acceleration of heavy ions up to the design beam energy. The required acceleration gain was achieved with heavy ion beams even above the design mass to charge ratio at high beam intensity and full beam transmission. This paper presents systematic beam measurements with varying rf amplitudes and phases of the $\mathrm{CH}$ cavity, as well as phase space measurements for heavy ion beams with different mass to charge ratio. The worldwide first and successful beam test with a superconducting multigap CH cavity is a milestone of the R\&D work of HIM and GSI in collaboration with IAP in preparation of the HELIAC project and other cw-ion beam applications.
\end{abstract}

DOI: 10.1103/PhysRevAccelBeams.21.020102

\section{INTRODUCTION}

An UNILAC upgrade program is ongoing, designated to prepare for high intensity high current heavy ion [1-5], as well as for proton $[6,7]$ synchrotron injector operation for the Facility of Antiproton and Ion Research (FAIR) [8]. As a result, high duty factor beam time availability for super heavy element (SHE) research at GSI Universal Linear Accelerator (UNILAC) will be strongly diminished due to the duty factor limitation for FAIR injector operation. Besides, an upgrade program of the High Charge State Injector (HLI) was already initialized comprising a new $18 \mathrm{GHz}$ electron cyclotron resonance ion source (ECR), a cw capable radio frequency quadrupole (RFQ) and an interdigital H-mode drift tube linac (IH-DTL) [9,10], keeping the SHE program at GSI competitive [11]. An additional standalone sc cw linac (HELIAC) is assumed to

\footnotetext{
*Corresponding author. w.barth@gsi.de

Published by the American Physical Society under the terms of the Creative Commons Attribution 4.0 International license. Further distribution of this work must maintain attribution to the author(s) and the published article's title, journal citation, and DOI.
}

meet the demands of the experimental program at its best. With significantly higher beam intensity the SHE production rate will be increased as well. HELIAC is based on superconducting $\mathrm{CH}$ cavities, efficient multicell structures combining the advantages of superconducting and long room temperature cavities [12].

The design and construction of cw high intensity linacs is a crucial goal of worldwide accelerator technology development [13-17]. Above all, compactness of a particle accelerator is a beneficial demand for the development of high intensity $\mathrm{cw}$ proton and ion linacs [18-20]. In the low- and medium-energy range cw linacs can be used for several applications, as boron-neutron capture therapy, high productivity isotope generation and material science. A highenergy linac is an integrated and essential part of several large scale research facilities, as spallation neutron sources or accelerator driven systems. Thus the study and investigation of the design, operation and optimization of a cw linac, as well as progress in elaboration of the superconducting technology [21] is of high relevance.

\section{GENERAL HELIAC LAYOUT AND R\&D}

Nine superconducting $\mathrm{CH}$ cavities operated at $217 \mathrm{MHz}$ provide for ion acceleration to beam energies between 3.5 


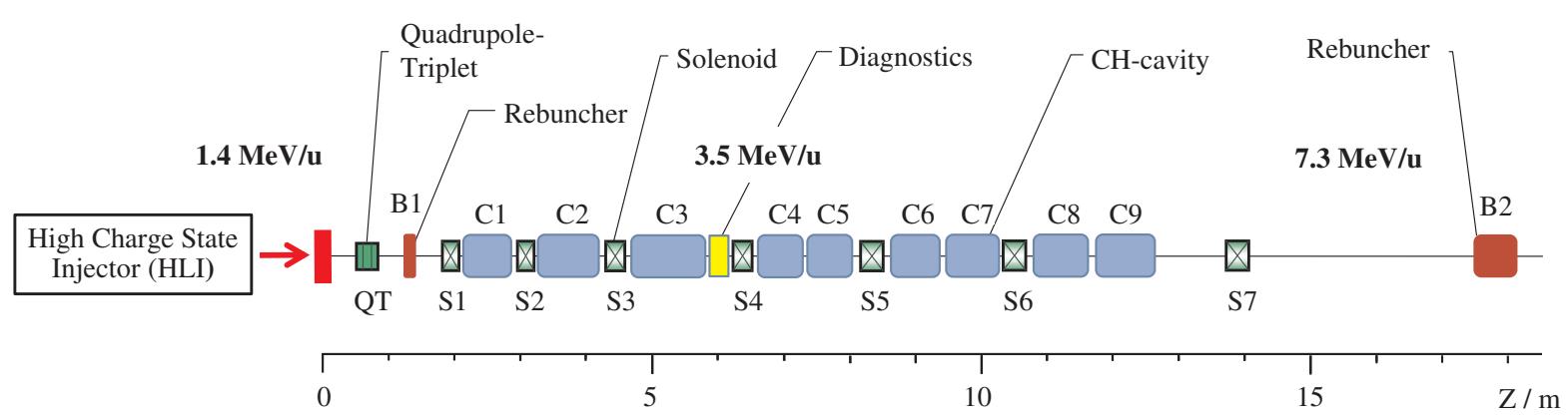

FIG. 1. General cw-linac layout.

and $7.3 \mathrm{MeV} / \mathrm{u}$, while the energy spread should be kept smaller than $\pm 3 \mathrm{keV} / \mathrm{u}$. A conceptual layout [22] (see Fig. 1) of this sc cw linac was worked out eight years ago. It allows the acceleration of highly charged ions with a mass to charge ratio of up to 6 . For proper beam focusing superconducting solenoids have to be mounted between the $\mathrm{CH}$ cavities. The general parameters are listed in Table I.

$R \& D$ and prototyping (demonstrator project) $[23,24]$ in preparation of the proposed HELIAC is assigned to a collaboration of GSI, HIM and IAP. The demonstrator setup, embedded in a new radiation protection cave, is located in the straightforward direction of the HLI (Fig. 2).

The liquid helium (LHe) supply is covered by a 30001 tank, while the consumed helium gas is collected in a $25 \mathrm{~m}^{3}$ recovery balloon and bottled by a compressor. The demonstrator [25] comprises a 15 gap sc $\mathrm{CH}$ cavity embedded by two superconducting solenoids; all three components are mounted on a common support frame $[26,27]$. The support frame, as well as the accelerator components, are suspended each by eight tie rods in a crosslike configuration balancing the mechanical stress during the cooldown and warm-up. The beam focusing solenoids provide maximum fields of $9.3 \mathrm{~T}$, the free beam aperture is $30 \mathrm{~mm}$. A configuration of one main coil out of $\mathrm{Nb}_{3} \mathrm{Sn}$ and two compensation coils made from $\mathrm{NbTi}$ shields the maximum magnetic field of $9.3 \mathrm{~T}$ within a longitudinal distance of $10 \mathrm{~cm}$ down to $30 \mathrm{mT}$. The solenoids are connected to LHe ports inside the cryostat by copper tapes allowing dry cooling. The sc $\mathrm{CH}$ structure (Fig. 3) is the key component and offers a variety of research and development.

TABLE I. Design parameters of the cw linac.

\begin{tabular}{lcc}
\hline \hline Mass/charge & & 6 \\
Frequency & $\mathrm{MHz}$ & 216.816 \\
Maximum beam current & $\mathrm{mA}$ & 1 \\
Injection energy & $\mathrm{MeV} / \mathrm{u}$ & 1.4 \\
Output energy & $\mathrm{MeV} / \mathrm{u}$ & $3.5-7.3$ \\
Output energy spread & $\mathrm{keV} / \mathrm{u}$ & \pm 3 \\
Length of acceleration & $\mathrm{m}$ & 12.7 \\
Sc CH cavities & $\mathrm{No}$ & 9 \\
Sc solenoids & $\mathrm{No}$. & 7 \\
\hline \hline
\end{tabular}

\section{A. Preparation and commissioning of the rf-cavity and rf-supply system}

The sc 15 gap $\mathrm{CH}$ cavity is directly cooled with liquid helium, supported by a helium jacket made by titanium. The vendor Research Instruments $\mathrm{GmbH}$ (RI) provided for sufficient cavity preparation. After high pressure rinsing (HPR) a performance test in a vertical cryostat at low rf power was performed at IAP, reaching gradients up to $7 \mathrm{MV} / \mathrm{m}$. After the final assembly of the helium vessel and further HPR preparation at RI, the cavity was tested again, but in a horizontal cryostat. The cavity showed improved performance due to an additional HPR treatment, the initial design quality factor $\mathrm{Q}_{0}$ has been exceeded by a factor of 4 , a maximum accelerating gradient of $\mathrm{E}_{\mathrm{acc}}=9.6 \mathrm{MV} / \mathrm{m}$ at $\mathrm{Q}_{0}=8.14 \times 10^{8}$ has been achieved [28,29]. Prior beam commissioning of the demonstrator cavity, the rf power couplers [30] were tested and conditioned with a dedicated test resonator [31] depicted in Fig. 4.

The couplers are equipped with sensors to control the temperature of the ceramic windows and the Langmuir probes to detect the multipacting current. A first conditioning [32] has been performed with pulsed rf (up to $5 \mathrm{~kW}$ ) and finally in cw mode (up to $2 \mathrm{~kW}$ ). Further increase of the forwarded cw-rf power leads to a temperatures rise of more than $80^{\circ} \mathrm{C}$ at the ceramic window, potentially sufficient to damage the coupler. During the operation, the "cold" coupler window has been anchored to the liquid nitrogen supply tube by copper ribbons. In a clean room of class ISO4 the power couplers were integrated in the rf cavity, as well as three frequency tuners, developed at IAP [33] and manufactured at GSI for the control of resonance frequency. Furthermore, the $\mathrm{CH}$ cavity and both solenoids were assembled on a string. After leak testing of the accelerating string the complete cold mass was integrated [34] into the cryostat outside of the clean room.

\section{MATCHING SECTION AND EQUUS BEAM DYNAMICS}

The beam dynamics layout behind the HLI at $1.4 \mathrm{MeV} / \mathrm{u}$ has been simulated in advance. In a preparing beam test run, it could be confirmed that the room temperature focusing quadrupoles (triplet and two duplets) and two 


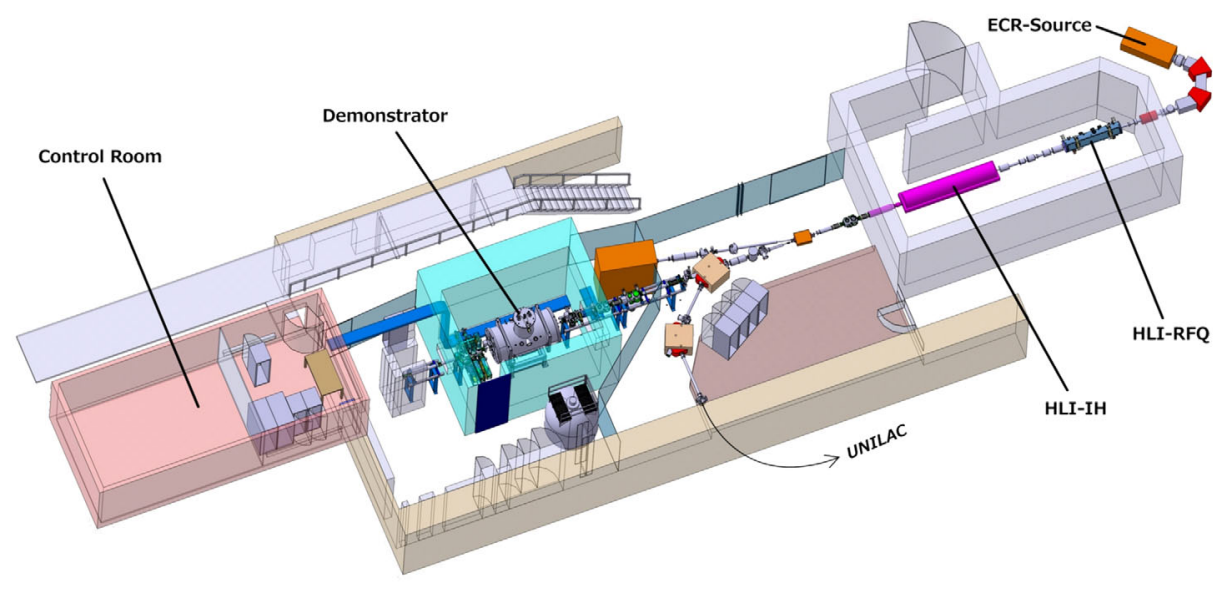

FIG. 2. CH-cavity test environment at GSI.

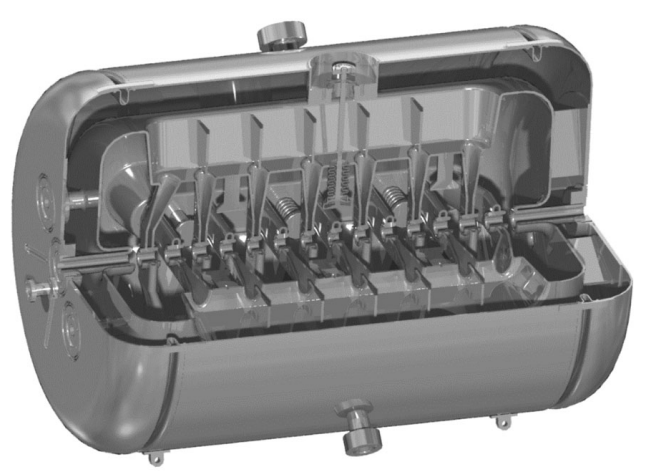

FIG. 3. Sectional drawing of the 15-gap demonstrator $\mathrm{CH}$ cavity.
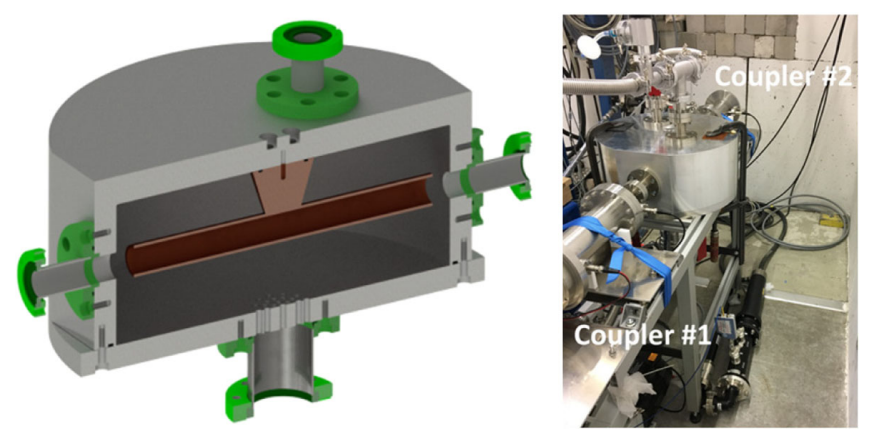

FIG. 4. Radio frequency testing cavity (left) for the rf power couplers at GSI; two couplers could be tested simultaneously (right) - the temperature at the outer coupler surface was measured with sensors. rebuncher cavities are sufficient to provide for full $6 \mathrm{D}$ matching to the demonstrator [35]. At the same time, the input beam is axially symmetric for further solenoid focusing due to especially chosen gradients, while bunch length and momentum spread is matched as well. The transport line (see Fig. 5) provides also for necessary beam diagnostics devices. Moreover, beam transformers, Faraday cups, SEM-profile grids, a dedicated emittance meter, a bunch structure monitor and phase probe pickups (beam energy measurements applying time of flight) provide for proper beam characterization behind demonstrator.

The beam dynamics layout of the sc cw linac is based on the equidistant multigap structure (EQUUS) concept, as proposed in [36]. It features high acceleration efficiency with longitudinal and transversal stability, as well as a straightforward energy variation. Energy variation can easily be achieved by varying the applied rf voltage or the rf phase of the amplifier. Highly charged ions with a mass-to-charge ratio of maximum 6 will be accelerated from 1.4 up to $3.5-7.3 \mathrm{MeV} / \mathrm{u}$. Energy variation while maintaining a high beam quality is the core issue with respect to beam dynamics, simulated using advanced software [37-39] and previously developed algorithms [40-43]. The cell length inside an EQUUS designed cavity is kept constant and is fixed with a higher (geometrical) $\beta$ compared to the injection beam energy (constant $\beta$ structure). As a consequence the constant $\beta$ structure leads to a sliding movement in longitudinal phase space. Trajectory and energy gain depend strongly on the initial phase at the first gap center and the difference between

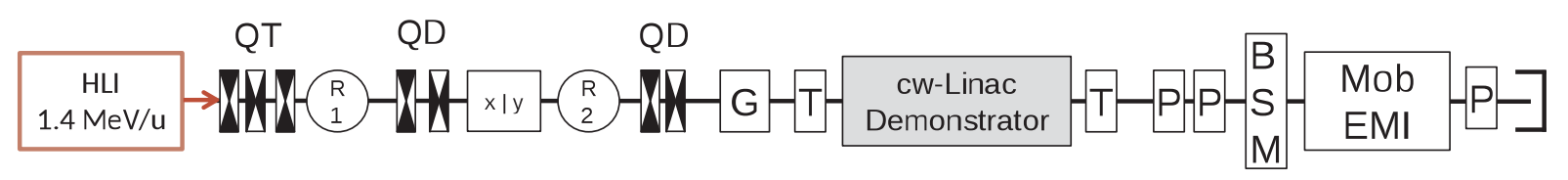

FIG. 5. Layout of matching line to the demonstrator and beam diagnostics test bench; QT $=$ quadrupole triplet, QD $=$ quadrupole duplet, $\mathrm{R}=$ rebuncher, $\mathrm{X} / \mathrm{Y}=$ beam steerer, $\mathrm{G}=\mathrm{SEM}$-grid, $\mathrm{T}=$ beam current transformer, $\mathrm{P}=$ phase probe, $\mathrm{BSM}=$ bunch shape monitor, $\mathrm{EMI}=$ emittance meter. 
particle energy and design energy. The corresponding transversal emittance evolution has been measured in a broad range with small emittance growth.

\section{BEAM MATCHING AND FIRST BEAM ACCELERATION}

For transverse matching an additional quadrupole doublet was installed in front of the demonstrator. Adequate

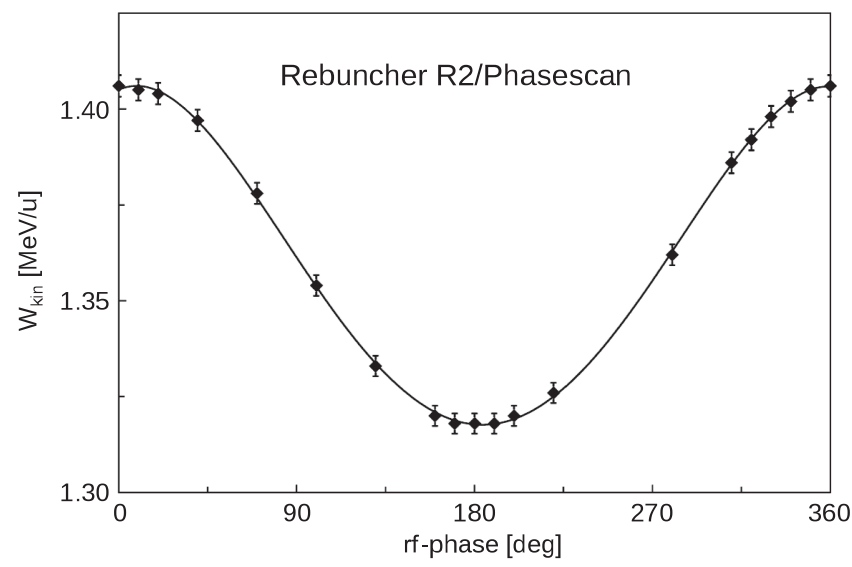

FIG. 6. $\mathrm{Ar}^{9+}$-rf phase scan with rebuncher R2; nominal beam energy (HLI exit) is measured for $1.366 \mathrm{MeV} / \mathrm{u}$.
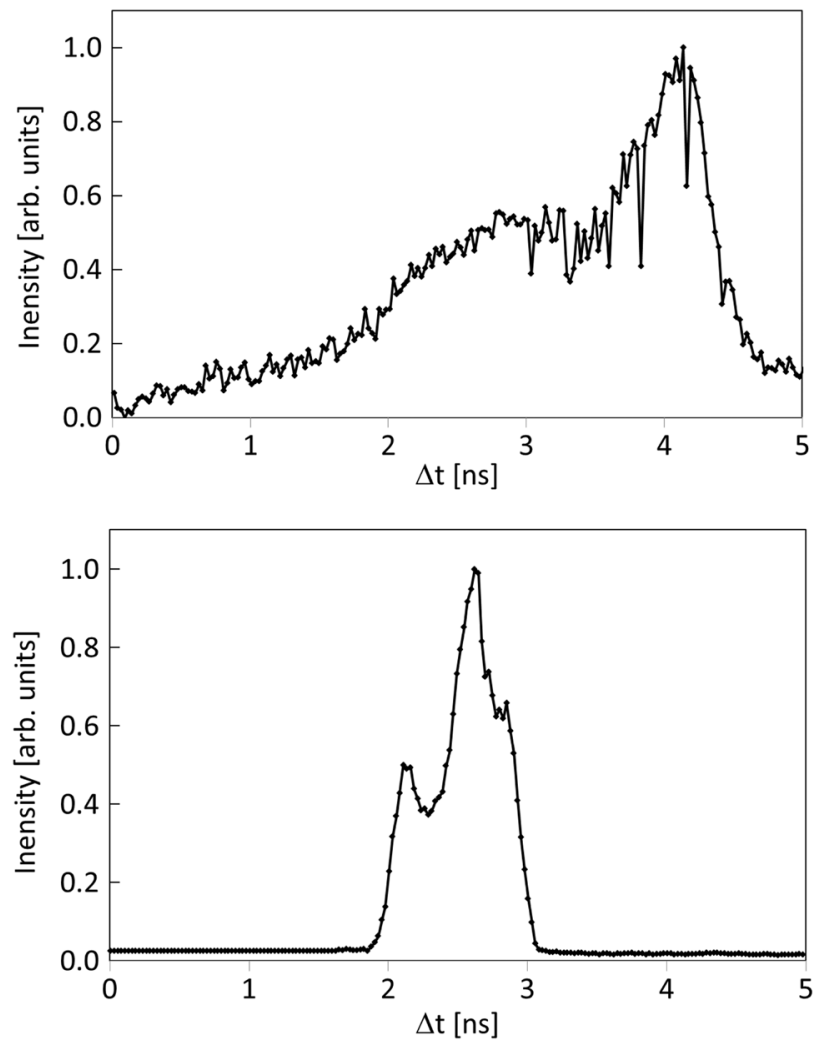

FIG. 7. Bunch shape measurement for HLI beam at $1.366 \mathrm{MeV} / \mathrm{u}$ (top) and at the same energy for the matched case with rebuncher R1 and R2 (bottom). longitudinal matching is accomplished by the existing rebuncher cavity behind HLI in combination with another newly built $108 \mathrm{MHz}$ quarter wave resonator (rebuncher) both with four rf gaps of the same construction [44]. Exemplary a measured $\mathrm{Ar}^{9+}$-phase scan (for nominal $\mathrm{rf}$ amplitude) of the second rebuncher R2 is shown in Fig. 6. The nominal bunching phase is reached at an rf phase of $+90^{\circ}$, where the HLI-output energy $(1.366 \mathrm{MeV} / \mathrm{u})$ is kept constant at a maximum longitudinal focusing effect.

For the measurement of the longitudinal bunch shape a Feschenko monitor [45] was installed behind the cryomodule. The bunch length at HLI output $(0.5 \mathrm{~ns}-0.8 \mathrm{~ns})$ is increased in the long drift line to the bunch shape monitor (see Fig. 7 at the top). For proper longitudinal matching to the demonstrator cavity rebuncher R1 provides for a parallel beam envelope. A longitudinal waste in the middle of the $\mathrm{CH}$ cavity is generated applying rebuncher $\mathrm{R} 2$. This condition was confirmed by shifting the beam focus until the measured bunch length at the monitor became the same as without R2. The measured bunch structure at the monitor for the matched case is shown in Fig. 7 (bottom), the bunch length at $\mathrm{R} 2$ and at the monitor is measured for $\approx 1 \mathrm{~ns}$ (base width).

On June 28, 2017, after successful rf testing of the superconducting rf cavity in 2016, setup of the matching line to the demonstrator and a short commissioning
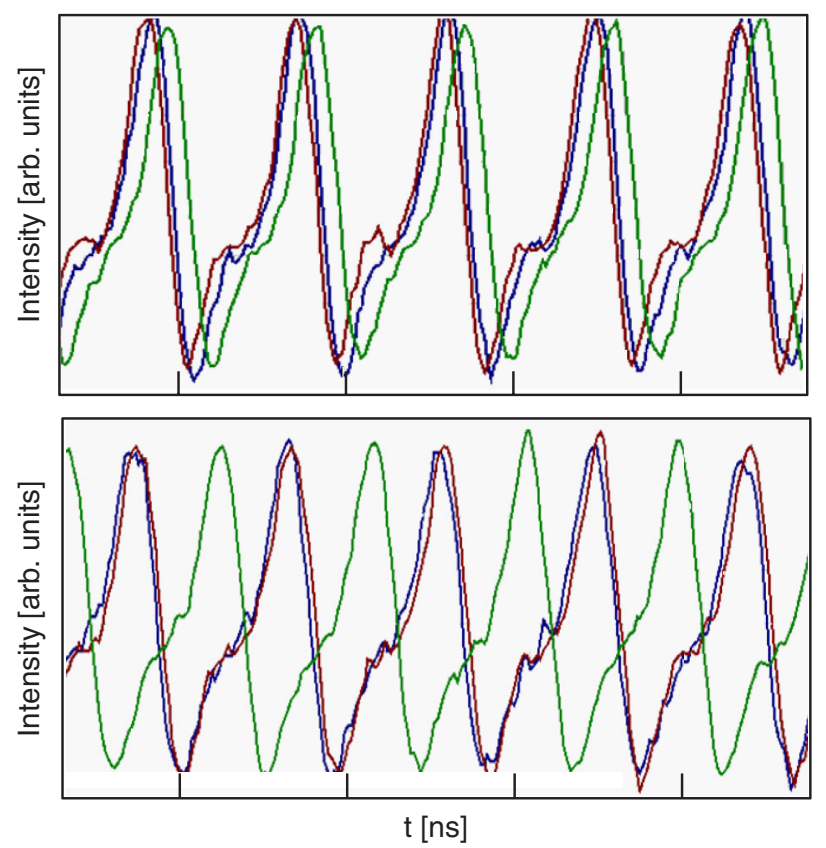

FIG. 8. First rf acceleration with $\mathrm{CH}$ cavity; measured $\mathrm{Ar}^{11+}$ phase probe signals from HLI beam at $1.366 \mathrm{MeV} / \mathrm{u}$ (top), rf frequency is $108.408 \mathrm{MHz}(\mathrm{T}=9.224 \mathrm{~ns})$. By acceleration up to the nominal beam energy (bottom), the coarse time of flight between the blue and the red signal is slightly reduced. The time of flight for the fine measurement between the red and the green signal is significantly shifted, according to the beam energy of $1.866 \mathrm{MeV} / \mathrm{u}$. 
TABLE II. Radio frequency parameters for matched case.

\begin{tabular}{lrrrr}
\hline \hline & $\mathrm{He}^{2+}$ & $\mathrm{Ar}^{11+}$ & $\mathrm{Ar}^{9+}$ & $\mathrm{Ar}^{6+}$ \\
\cline { 2 - 5 } $\mathrm{A} / \mathrm{q}$ & 2.0 & 3.6 & 4.4 & 6.7 \\
\hline $\mathrm{U}_{\text {Reb1,eff }}{ }^{\mathrm{a}}[\mathrm{kV}]$ & 8.3 & 15.0 & 18.3 & 27.9 \\
$\mathrm{U}_{\text {Reb2,eff }}[\mathrm{kV}]$ & 22.7 & 40.8 & 49.9 & 75.9 \\
$\mathrm{E}_{\mathrm{acc}, \mathrm{CH}}{ }^{2}[\mathrm{MV} / \mathrm{m}]$ & 1.8 & 3.2 & 3.9 & 5.9 \\
$\mathrm{U}_{0}[\mathrm{MV}]$ & 1.2 & 2.2 & 2.7 & 4.0 \\
\hline \hline
\end{tabular}

${ }^{\mathrm{a}}$ Referring to voltage calibration with measured beam energy (see Fig. 6).

${ }^{\mathrm{b}} E_{\text {acc }}=$ transit time factor $\times$ total accelerating voltage/ $(n \times 0.5 \cdot \beta \lambda)$.

and ramp-up time of some days, the $\mathrm{CH}$ cavity accelerated heavy ion beams $\left(\mathrm{Ar}^{1+}\right)$ with full transmission for the first time up to the design beam energy of $1.866 \mathrm{MeV} / \mathrm{u}$ $\left(\Delta \mathrm{W}_{\text {kin }}=0.5 \mathrm{MeV} / \mathrm{u}\right)$. In Fig. 8, the measured phase probe signals from HLI beam at $1.366 \mathrm{MeV} / \mathrm{u}$ (top) is shown; the rf frequency is $108.408 \mathrm{MHz}(\mathrm{T}=9.224 \mathrm{~ns})$. By acceleration up to the nominal beam energy (bottom), the coarse time of flight between the blue and the red signal is slightly reduced. The time of flight for the fine measurement between the red and the green signal is significantly increased, according to the beam energy of $1.866 \mathrm{MeV} / \mathrm{u}$.

For the first beam test the superconducting cavity was powered with 10 Watt of net rf power providing an accelerating voltage of more than 1.6 MV inside a length of $69 \mathrm{~cm}$. Further on the design acceleration gain of $3.5 \mathrm{MV}$ has been verified and even exceeded by acceleration of heavy ion beam with high rigidity $(\mathrm{A} / \mathrm{q}=6.7)$. As summarized in Table II argon and helium ion beam with different charge state from an ECR ion source $\left({ }^{4} \mathrm{He}^{2+}\right.$, ${ }^{40} \mathrm{Ar}^{11+},{ }^{40} \mathrm{Ar}^{9+},{ }^{40} \mathrm{Ar}^{6+}$ ) were accelerated at HLI for further beam tests with the demonstrator. For longitudinal beam matching the rebuncher (R1, R2) settings were adapted according to the mass of charge ratio $\mathrm{A} / \mathrm{q}$, as well as the acceleration voltage $\mathrm{U}$.

A maximum average beam intensity of $1.5 \mathrm{p} \mu \mathrm{A}$ has been achieved, limited only by the pulse intensity of the injector and its maximum duty factor (25\%), while the $\mathrm{CH}$ cavity was operated in $\mathrm{cw}$ mode. All the presented measurement results were accomplished with high duty factor beam and maximum beam intensity available from the HLI.

\section{SYSTEMATIC AMPLITUDE-PHASE SCANS}

Systematic parameter studies and detailed investigations of beam behavior were started after basic operational capability and reliability of all rf cavities, rf-supply systems, cryosupply and all other subsystems were accomplished. Besides heavy ion beam operation of the ECR ion source, low energy beam transport line and HLI-RFQ, IH-DTL and matching line to the demonstrator could be set up for maximum stable and reliable operation during the entire machine investigation time. Readjustment of the

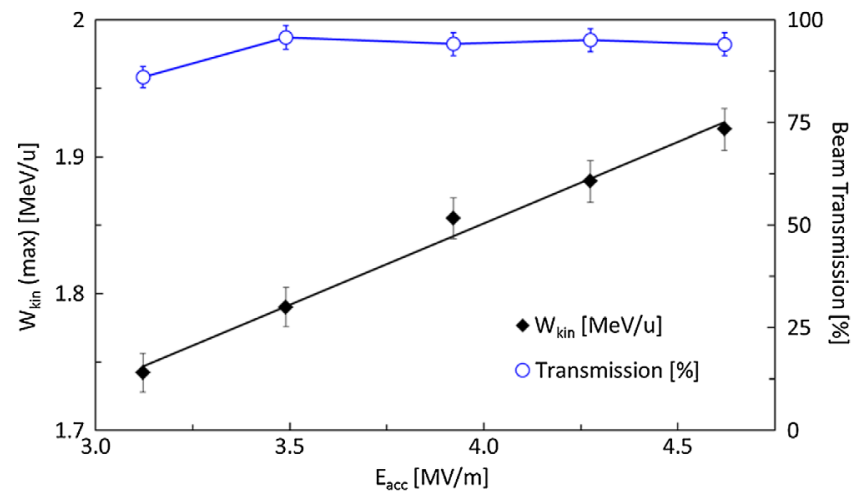

FIG. 9. Acceleration of an $\mathrm{Ar}^{9+}$ beam; maximum achieved beam energy and transmission as a function of the accelerating gradient.

complex accelerator system for operation with different ion species was achieved inside less than four hours until beam investigation could be continued. Mainly the refill with liquid helium from the Dewar led to serious interruptions of the beam investigations program. By all means, each of the presented measurements could be achieved and finalized nonstop.

The initial amplitude scan (rf-phase $=30^{\circ}$ ) with $\mathrm{Ar}^{9+}$ beam is shown in Fig. 9. For accelerating gradients between 3.1 and $4.6 \mathrm{MV} / \mathrm{m}$ the beam energy scales linearly with a gradient of $0.12 \mathrm{MeV} / \mathrm{u}$ per $1 \mathrm{MV} / \mathrm{m}$ (for $\mathrm{Ar}^{9+}$ ). Upscaling to the design energy gain $\left(\Delta \mathrm{W}_{\text {kin }}=0.52 \mathrm{MeV} / \mathrm{u}, \mathrm{A} / \mathrm{q}=6\right)$ leads to a nominal (effective) accelerating gradient of $(5.9 \pm 0.05) \mathrm{MV} / \mathrm{m}$, slightly above the design gradient of $5.5 \mathrm{MV} / \mathrm{m}$. In any case the capability in terms of the accelerating gradient is even high enough to fulfill the design criterion. The measured beam transmission is $(95 \pm 1) \%$, except for low accelerating gradients. The reason for this drop is a slight transversal overfocusing of the beam due to the missing rf defocusing at lower rf gradient. This transmission drop is generally easy to compensate by readjusting the magnetic quadrupole and/or solenoid field setting.

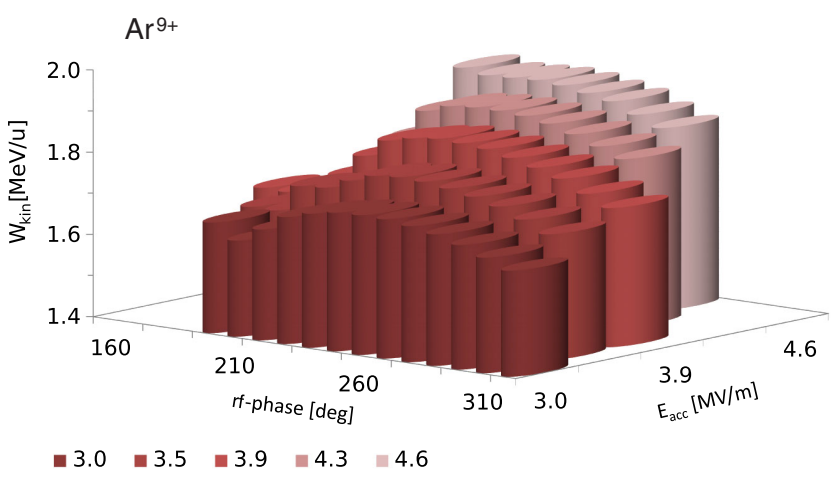

FIG. 10. 3D scan of $\mathrm{Ar}^{9+}$-beam energy versus accelerating gradient and $\mathrm{rf}$ phase. 


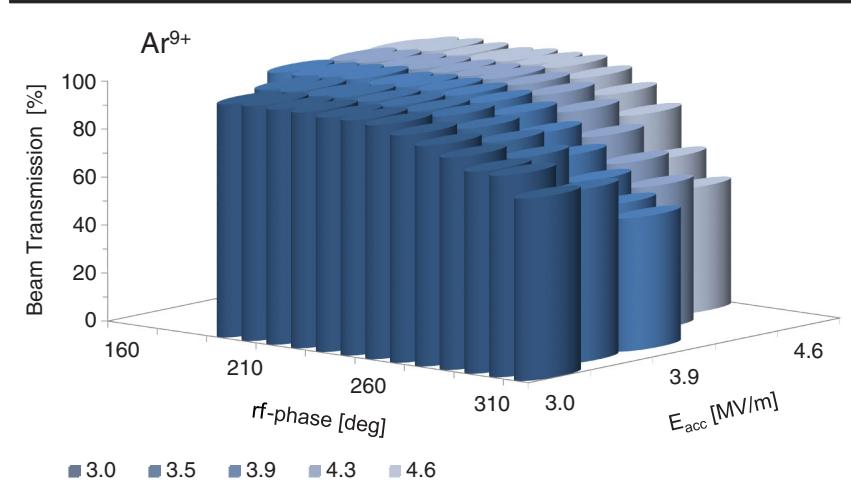

FIG. 11. 3D scan of $\mathrm{Ar}^{9+}$-beam transmission versus accelerating gradient and rf phase.

In Figs. 10 and 11 a full measured 3D scan of beam energy and beam transmission for a wide area of different accelerating fields and rf phases is depicted. As in Fig. 10 the linear increase of beam energy with ramped accelerating gradient could be observed for different rf phase settings, while the beam transmission is kept above $90 \%$. To gain for the maximum beam energy at a given accelerating gradient the rf phase has to be adapted slightly. In general these measurements confirm impressively the EQUUS beam dynamics, featuring effectively the nonresonant beam acceleration up to different beam energies without particle loss and significant beam quality degradation. As measured with helium beam and recently confirmed by beam dynamics simulations, for lighter ions maximum beam energy of up to $2.2 \mathrm{MeV} / \mathrm{u}$ could be reached with the demonstrator cavity, with reduced beam quality.

The bunch length detected with the bunch shape monitor (BSM) was measured as very sensitive to rf-phase changes. As shown in Figs. 12 and 13, a change of rf phase by $30^{\circ}$ only, leads to a change of bunch length by more than a factor of 4, while the beam transmission is not effected. For further matching to another $\mathrm{CH}$ cavity, as planned for the new cw-linac project (HELIAC), the adjustment of the beam energy setting by changing the rf amplitude is more

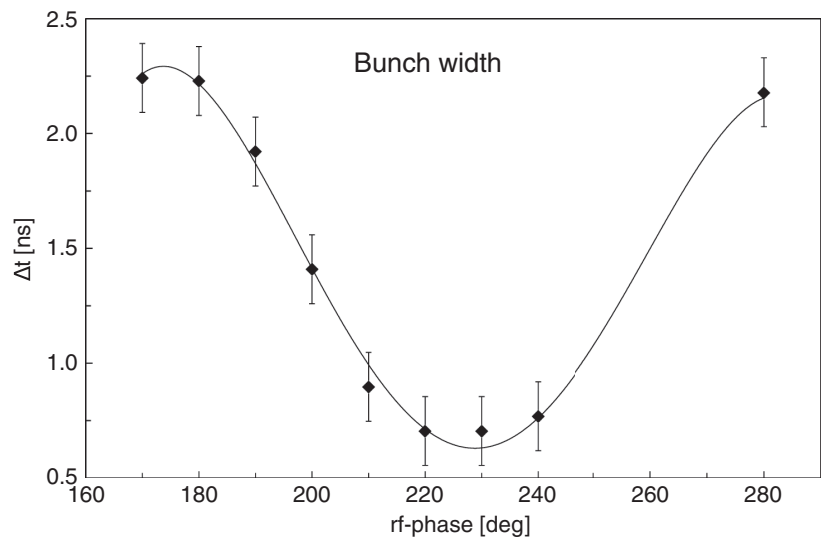

FIG. 12. Measured width of the bunch length ( $r$ phase scan) for $\mathrm{Ar}^{9+}$ beam at an accelerating gradient of $3.5 \mathrm{MV} / \mathrm{m}$.
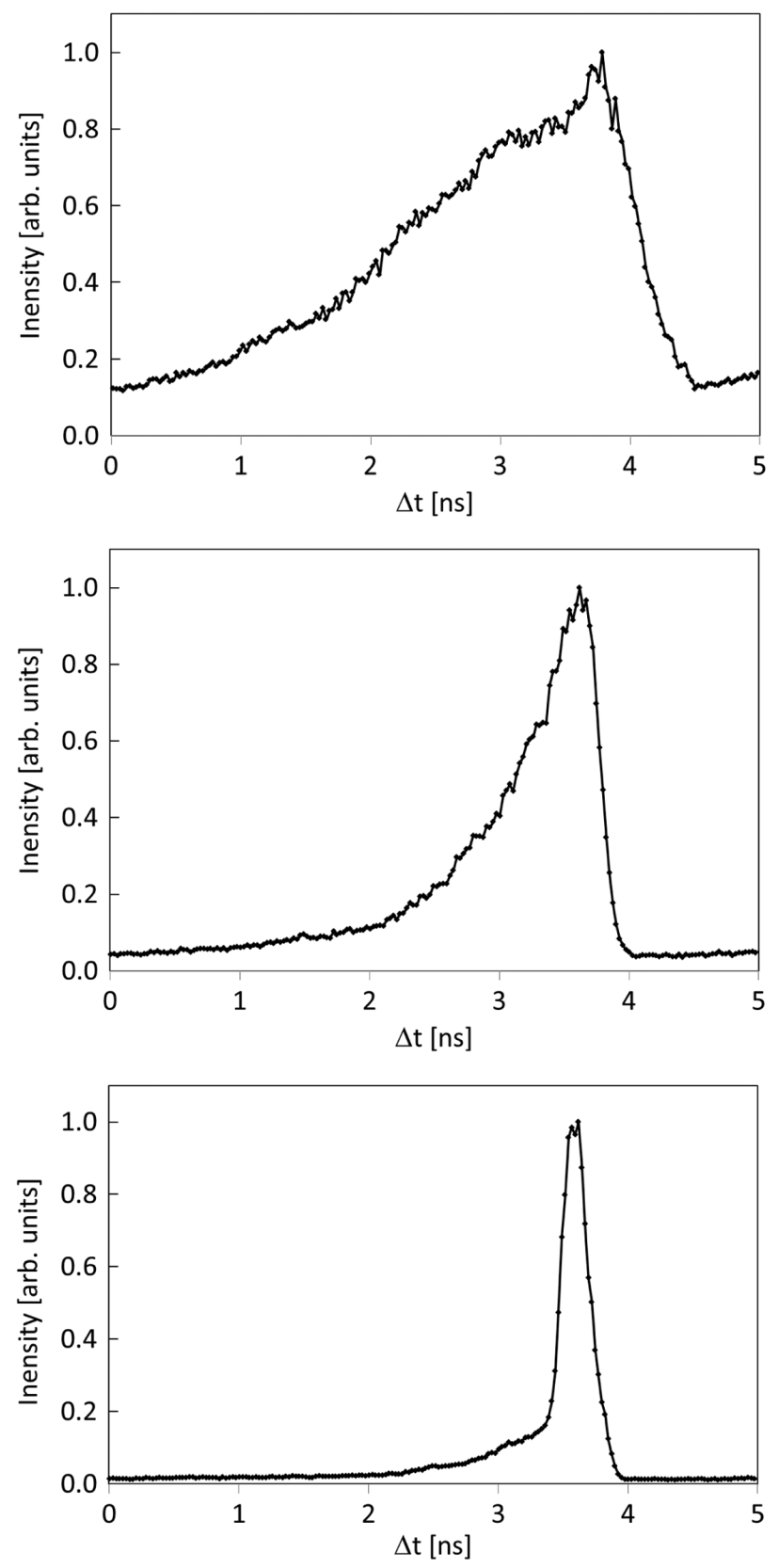

FIG. 13. Selected bunch structure for $\mathrm{Ar}^{9+}$ at a gradient of $3.5 \mathrm{MV} / \mathrm{m}$ for an $\mathrm{rf}$ phase of $190^{\circ}, 200^{\circ}$ and $220^{\circ}$ according to Fig. 12.

favorable-compared to changing the rf phase-as no significant bunch shape change could be observed.

For heavy ion beams above the design mass over charge ratio $(\mathrm{A} / \mathrm{q}=6)$ acceleration up to the nominal beam energy were achieved. With $\mathrm{Ar}^{6+}$ beam $(\mathrm{A} / \mathrm{q}=6.7)$, an energy gain above $0.5 \mathrm{MeV} / \mathrm{u}$ could be reached with an accelerating gradient of $6 \mathrm{MV} / \mathrm{m}$. As an example Fig. 14 shows a fully measured $360^{\circ}$ phase scan for two different accelerating gradients $(3.5$ and $5.5 \mathrm{MV} / \mathrm{m})$. All individual data as well as the characteristic shapes of the phase scans are in good agreement according to the accelerating gradient. For 


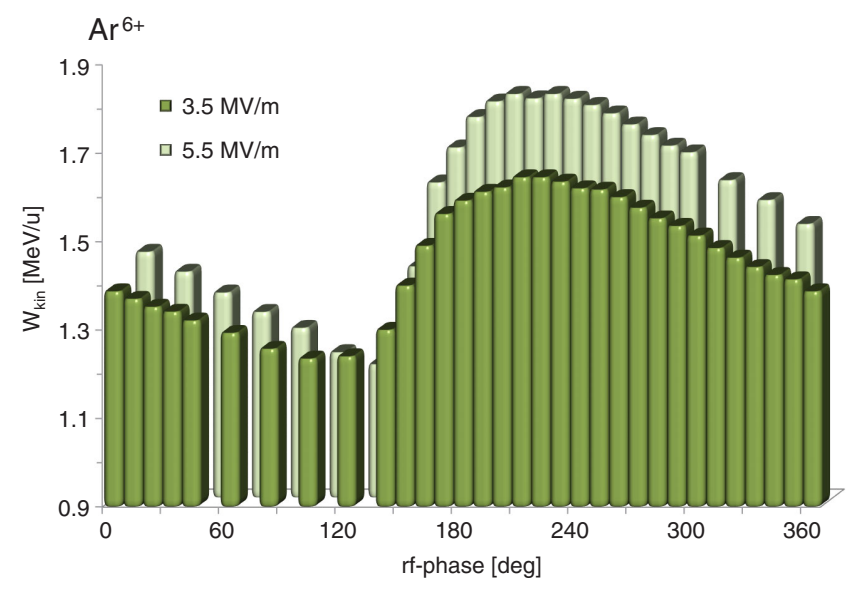

FIG. 14. Phase scan of $\mathrm{Ar}^{6+}$-beam energy for $3.5 \mathrm{MV} / \mathrm{m}$ and $5.5 \mathrm{MV} / \mathrm{m}$.

an increased gradient the maximum beam energy at an $\mathrm{rf}$ phase of $210^{\circ}$ boosts as well, while the minimum beam energy at $130^{\circ}$ could be decreased down to $1.2 \mathrm{MeV} / \mathrm{u}$.

In Fig. 15 the measured $\mathrm{Ar}^{6+}$-phase scan of beam energy and beam transmission is depicted with more steps in $\mathrm{rf}$ phase. A high beam transmission is confirmed in a wide phase range $\left(0^{\circ} \leq \mathrm{rf}\right.$ phase $\left.\leq 230^{\circ}\right)$. In this phase range for $\mathrm{Ar}^{6+}$ beam the beam energy could be changed by $\Delta \mathrm{W}_{\text {kin }}=$ $0.7 \mathrm{MeV} / \mathrm{u}$ without transmission degradation. For lighter heavy ion beams (e.g. ${ }^{4} \mathrm{He}^{2+}$ ) a full beam energy swing of $1 \mathrm{MeV} / \mathrm{u}$ could be reached without beam transmission drop.

At maximum of the measured phase scan of beam energy $\left(210^{\circ}\right.$ in Fig. 15) an amplitude scan has been performed as well (Fig. 16). For this measurement the rf phase is changed in a wide range of accelerating gradients $\left(0 \mathrm{MV} / \mathrm{m}<\mathrm{E}_{\mathrm{acc}}<6 \mathrm{MV} / \mathrm{m}\right)$. As a result the beam energy saturates with increased accelerating gradient, limiting strongly the energy gain. The maximum beam energy is achievable, if the rf amplitude is ramped up and the rf phase is set to the local beam energy maximum. No beam transmission degradation has been observed for the full

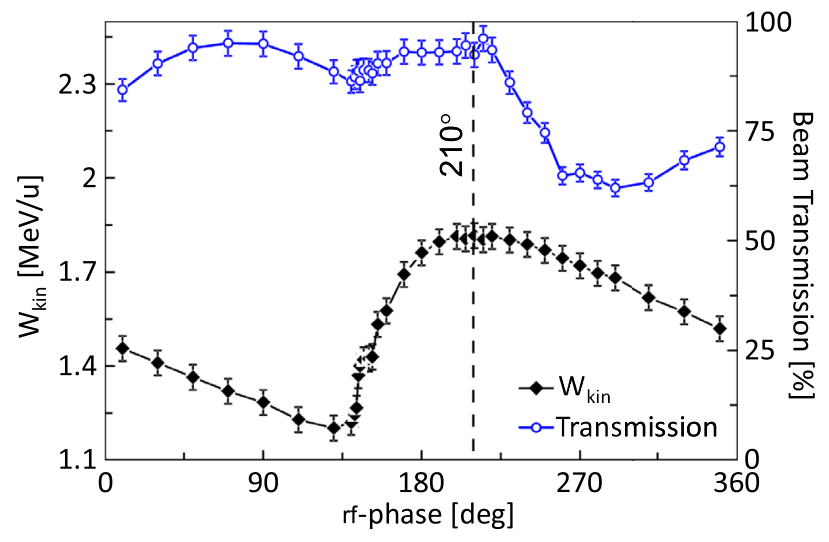

FIG. 15. Phase scan of $\mathrm{Ar}^{6+}$-beam energy for $5.5 \mathrm{MV} / \mathrm{m}$.

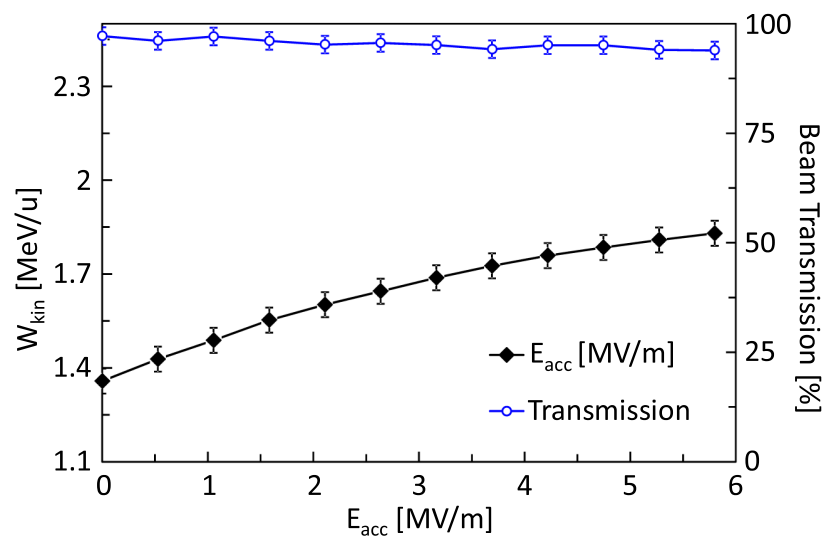

FIG. 16. Amplitude scan of $\mathrm{Ar}^{6+}$-rf phase of $210^{\circ}$.

range of $\mathrm{rf}$ amplitudes, while the focusing strengths of quadrupole lenses were adapted.

\section{PHASE SPACE MEASUREMENTS}

Finally, the beam quality has been characterized by measuring the covered area in phase space exemplary with $\mathrm{Ar}^{9+}$ beam. The measured emittance of the argon beam, delivered by the ECR and HLI, shows a good beam quality: The total $90 \%$ horizontal beam emittance is measured for
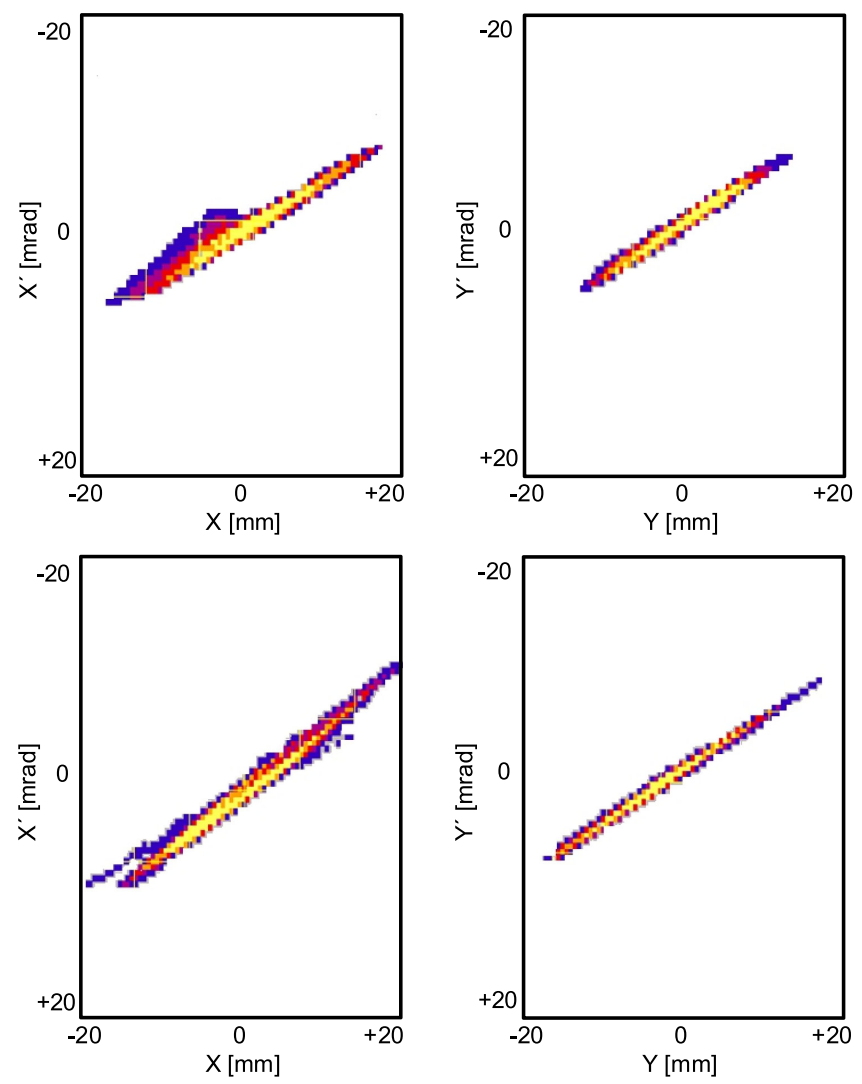

FIG. 17. Transverse $\mathrm{Ar}^{9+}$-beam emittance at $1.366 \mathrm{MeV} / \mathrm{u}$ (top) and at $1.85 \mathrm{MeV} / \mathrm{u}$ (bottom); normalized (horizontal) emittance growth is measured for $15 \%$. 

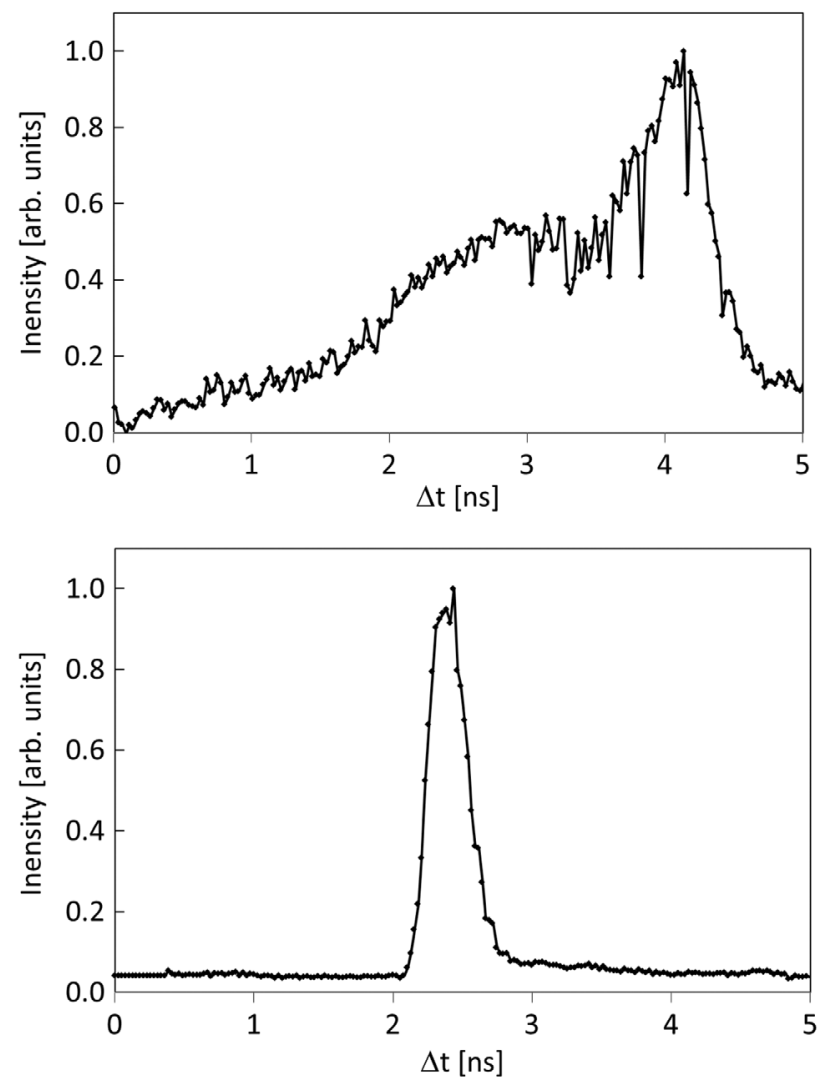

FIG. 18. Bunch shape of $\mathrm{Ar}^{9+}$-beam emittance at $1.366 \mathrm{MeV} / \mathrm{u}$ (top) and fully matched at $1.85 \mathrm{MeV} / \mathrm{u}$ (bottom); the FWHM of is measured for $0.5 \mathrm{~ns}$.

$0.74 \mu \mathrm{m}$, while in the vertical plane the total $90 \%$ emittance is $0.47 \mu \mathrm{m}$ only (Fig. 17, at the top). The beam emittance for the matched case measured at nominal beam energy of $1.85 \mathrm{MeV} / \mathrm{u}$ is shown in Fig. 17 (bottom). All measurements have been performed without solenoidal field, therewith any additional emittance degradation effects by different beam focusing could be avoided. The measured (normalized) beam emittance growth at full beam transmission is low: $15 \%$ (horizontal plane) and 10\% (vertical plane). Selective measurements at other rf amplitudes and phases, as well as for other beam rigidities, confirmed the high (transversal) beam performance in a wide range of different parameters.

Besides beam energy measurements the bunch shape was measured after the matching procedure is accomplished (see Fig. 7) with the Feschenko monitor. As shown in Fig. 18 an impressive small minimum bunch length of about 300 ps (FWHM) and 500 ps (base) could be detected, sufficient for further matching to and acceleration in future rf cavities.

\section{SUMMARY AND OUTLOOK}

Generally, the proposed cw linac should facilitate variable output energy from 3.5 to $7.3 \mathrm{MeV} / \mathrm{u}$. The achieved beam commissioning of the demonstrator is a major milestone paving the way to the entire $\mathrm{cw}$ linac (HELIAC). The demonstrator set up, as the first section of a cw linac reached acceleration of heavy ions up to the design beam energy gain of $0.52 \mathrm{MeV} / \mathrm{u}$. The design acceleration gain was achieved with heavy ion beams even above the design mass to charge ratio at full transmission and high beam intensity ( $1.5 \mathrm{p} \mu \mathrm{A}$ at $25 \%$ beam duty). The beam duty is limited only by the HLI performance, while the cavity was operated in cw mode-after upgrade of HLI a beam intensity of $6 \mathrm{p} \mu \mathrm{A}$ is potentially achievable with the demonstrator cavity. At full beam transmission the beam quality was measured as excellent in a wide range of different beam energies, confirming the capabilities of the applied nonresonant beam dynamics design (EQUUS). For lighter heavy ions a maximum energy variation of $1 \mathrm{MeV} / \mathrm{u}$ was achieved without significant performance degradation.

Recently the possibility for acceleration of protons as well as uranium ion beams is under investigation [46]. A higher acceleration gradient for the first $\mathrm{sc} \mathrm{cw}-\mathrm{CH}$ cavity was confirmed in the presented experiments and has to be taken into account for further studies $[47,48]$. Therefore the original linac layout has to be revised by decreasing the number of gaps per cavity, preserving the total cavity voltage and high accelerating gradient. The maximum beam energy, which could be potentially reached by an advanced linac layout, has been estimated for ions with different mass to charge ratios $(1 \leq \mathrm{A} / \mathrm{Z} \leq 6)$. Moreover the effective acceleration of uranium ion beams $(\mathrm{A} / \mathrm{q}=8.5)$ is potentially achievable. Taking the presented encouraging results from the recent experimental campaign into account, the cw-linac HELIAC is at the top of recent accelerator R\&D activities. Therefore a strong interest for the accelerator community has to be stated.

\section{ACKNOWLEDGMENTS}

Successful beam testing could not be accomplished without strong support of highly committed people from different GSI departments. The beam test is a milestone of the R\&D work of HIM and GSI in collaboration with IAP in preparation of a superconducting heavy ion continuous wave linear accelerator.

[1] W. Barth et al., $\mathrm{U}^{28+}$-intensity record applying a $\mathrm{H}_{2}$-gas stripper cell, Phys. Rev. ST Accel. Beams 18, 040101 (2015).

[2] A. Adonin and R. Hollinger, Beam brilliance investigation of high current ion beams at GSI heavy ion accelerator facility, Rev. Sci. Instrum. 85, 02 A727 (2014).

[3] L. Groening et al., Experimental Evidence of Space Charge Driven Emittance Coupling in High Intensity Linear Accelerators, Phys. Rev. Lett. 103, 224801 (2009).

[4] W. Barth, W. Bayer, L. Dahl, L. Groening, S. Richter, and S. Yaramyshev, Upgrade program of the high current heavy 
ion UNILAC as an injector for FAIR, Nucl. Instrum. Methods Phys. Res., Sect. A 577, 211 (2007).

[5] L. Groening et al., Benchmarking of measurement and simulation of transverse rms-emittance growth, Phys. Rev. ST Accel. Beams 11, 094201 (2008).

[6] A. Adonin, W. Barth, F. Heymach, R. Hollinger, H. Vormann, and A. Yakushev, Production of high current proton beams using complex H-rich molecules at GSI, Rev. Sci. Instrum. 87, 02B709 (2016).

[7] W. Barth, A. Adonin, S. Appel, P. Gerhard, M. Heilmann, F. Heymach, R. Hollinger, W. Vinzenz, H. Vormann, and $\mathrm{S}$. Yaramyshev, Heavy ion linac as a high current proton beam injector, Phys. Rev. ST Accel. Beams 18, 050102 (2015).

[8] W. Barth et al., High brilliance uranium beams for the GSI FAIR, Phys. Rev. ST Accel. Beams 20, 050101 (2017).

[9] W. Barth et al., UNILAC-upgrade program for the heavy element research at GSI-SHIP, in Proceedings of the 10th European Particle Accelerator Conference, Edinburgh, Scotland, 2006 (EPS-AG, Edinburgh, Scotland, 2006) pp. 1565-1567.

[10] P. Gerhard et al., Commissioning of a new cw radio frequency quadrupole at GSI, in Proceedings of the International Particle Accelerator Conference, Kyoto, Japan (ICR, Kyoto, 2010), pp. 741-743.

[11] S. Hofmann et al., Heavy element research at GSI-SHIP, in Proceedings of International Symposium on Exotic Nuclei EXON'04, Peterhof, Russia, 2004 (Penionzhkevich, Yuri Erastovich Cherepanov, Evgeni A, 2004), pp. 3-20.

[12] H. Podlech, U. Ratzinger, H. Klein, C. Commenda, H. Liebermann, and A. Sauer, Superconducting CH structure, Phys. Rev. ST Accel. Beams 10, 080101 (2007).

[13] C. Prior, Overview of high intensity accelerator projects, in Proceedings of the 46th ICFA Advanced Beam Dynamics Workshop on High-Intensity and High Brightness Hadron Beams HB2010, Morschach, Switzerland, 2010 (PSI, Viligen, Switzerland, 2011), pp. 6-10.

[14] T. V. Kulevoy et al., Modeling of proton beam dynamics in an accelerator-driver at 600-1000 MeV and investigation of the electrodynamic characteristics of accelerating cavities, At. Energ. 117, 347 (2015).

[15] P. N. Ostroumov, Advances in CW ion linacs, in Proceedings of IPAC'15 Conference, Richmond, VA, USA (2015), pp. 4085-4090.

[16] N. Solyak et al., The concept design of the CW linac of the Project X, in Proceedings of the International Particle Accelerator Conference, Kyoto, Japan (ICR, Kyoto, 2010), pp. 654-656.

[17] A. V. Butenko et al., Development of NICA injection complex, in Proceedings of IPAC'14 Conference, Dresden, Germany (2014), pp. 2103-2105.

[18] S. M. Polozov et al., Beam dynamics study and electrodynamics simulations for the cw RFQ, in Proceedings of IPAC2017 Conference, Copenhagen, Denmark (2017), pp. 1333-1336.

[19] L. Weissman, D. Berkovits et al., SARAF Phase I linac operation in 2013-2014, J. Instrum. 10, T10004 (2015).

[20] M. A. Gusarova et al., Research and design of a new RFQ injector for modernization of the LU-20 drift-tube linac, Phys. Part. Nucl. Lett. 13, 915 (2016).
[21] R. Laxdal, Recent progress in the superconducting RF Program at TRIUMF/ISAC, Physica (Amsterdam) 441C, 13 (2006).

[22] S. Minaev, U. Ratzinger, H. Podlech, M. Busch, and W. Barth, Superconducting, energy variable heavy ion linac with constant $\beta$, multicell cavities of CH-type, Phys. Rev. ST Accel. Beams 12, 120101 (2009).

[23] S. Mickat et al., The sc cw-linac demonstrator-SRF technology finds the way to GSI, in Proceedings of SRF'11 Conference, Chicago, USA (2011), pp. 646-649.

[24] V. Gettmann et al., The sc cw-Linac Demonstrator - First section of a sc cw-Linac, Proceedings of $S R F^{\prime} 11$ Conference, Chicago, USA (2011), pp. 144-146.

[25] S. Mickat et al., Status of the superconducting cw Linac at GSI, in Proceedings of EXON'12, Vladivostok, Russia (2013), pp.337-346, DOI: 10.1142/9789814508865_0044.

[26] V. Gettmann et al., Status of the sc cw-linac demonstrator, in Proceedings of SRF'13 Conference, Paris, France (2013), pp. 80-82.

[27] V. Gettmann et al., Recent status new superconducting cw heavy ion Linac@GSI, in Proceedings of SRF'15 Conference, Whistler, Canada (2015), pp. 589-591.

[28] F. Dziuba et al., First performance test on the superconducting $217 \mathrm{MHz} \mathrm{CH}$ cavity at $4.2 \mathrm{~K}$, in Proceedings of LINAC'16 Conference, East Lansing, MI, USA (2016), pp. 953-955.

[29] F. Dziuba et al., First cold tests of the superconducting cw demonstrator at GSI, in Proceedings of RuPAC'16 Conference, St. Petersburg, Russia (2016), pp. 84-86.

[30] M. Heilmann et al., High power rf coupler for the cw-linac demonstrator at GSI, in Proceedings of IPAC'17, Copenhagen, Denmark (2017), pp. 990-992.

[31] M. Busch et al., Update on the sc $325 \mathrm{MHz} \mathrm{CH}$-cavity and power coupler processing, in Proceedings of LINAC'16, East Lansing, MI, USA (2017), pp. 913-915.

[32] F. Dziuba et al., Performance tests of the superconducting $217 \mathrm{MHz} \mathrm{CH}$ cavity for the cw Demonstrator, in Proceedings of SRF'17 Conference, Lanzhou, China (2017), p. TUPB024.

[33] M. Amberg et al., The fast piezo-based frequency tuner for sc CH-cavities, in Proceedings of LINAC'14 Conference, Geneva, Switzerland (2014), pp. 214-216.

[34] V. Gettmann et al., Commissioning of a superconducting cw heavy ion Linac@GSI, in Proceedings of SRF'17 Conference, Lanzhou, China (2017), p. MOPB094.

[35] M. Schwarz et al., Beam dynamics for the sc cw heavy ion Linac at GSI, in Proceedings of IPAC'15 Conference, Richmond, VA, USA (2015), pp. 3742-3744.

[36] M. Miski-Oglu et al., Steps towards superconducting cw linac for heavy ions at GSI, in Proceedings of SRF'17 Conference, Lanzhou, China (2017), p. MOPB024.

[37] R. Tiede et al., LORASR code development, in Proceedings of the 10th European Particle Accelerator Conference, Edinburgh, Scotland, 2006 (EPS-AG, Edinburgh, Scotland, 2006), pp. 2194-2196.

[38] S. Yaramyshev, W. Barth, L. Groening, A. Kolomiets, and T. Tretyakova, Development of the versatile multi-particle code DYNAMION, Nucl. Instrum. Methods Phys. Res., Sect. A 558, 90 (2006). 
[39] K. R. Crandall and D. P. Rusthoi, Report No. LA-UR-97886, 1997.

[40] S. Yaramyshev et al., An advanced procedure for longitudinal beam matching for sc $\mathrm{cw}$ heavy ion linacs, in Proceedings of HB'16, Malmo, Sweden (2016), pp. 571-574.

[41] M. Schwarz et al., Beam dynamics simulations for the new superconducting cw heavy ion linac at GSI, in Proceedings of SRF'17, Lanzhou, China (2017), p. MOPB005.

[42] S. M. Polozov et al., Beam dynamics simulations and code comparison for a new cw RFQ design, in Proceedings of HB'16, Malmo, Sweden (2016), pp. 188-191.

[43] S. Yaramyshev et al., Virtual charge state separator as an advanced tool coupling measurements and simulations, Phys. Rev. ST Accel. Beams 18, 050103 (2015).
[44] A. Orzhekhovskaya et al., Status report of the beam dynamics simulations for cw demonstrator, Annual Report Helmholtz Institute Mainz (HIM), 2012.

[45] A. V. Feschenko, Technique and instrumentation for bunch shape measurements, in Proceedings of RuPAC'12 Conference, Saint-Petersburg, Russian Federation (2012), pp. 181-185.

[46] S. Yaramyshev et al., Beam dynamics study for the HIM/ GSI heavy ion sc cw-Linac, in Proceedings of IPAC'17 Conference, Copenhagen, Denmark (2017), pp. 2217-2220.

[47] W. Barth et al., Further layout investigations for a superconducting cw-linac for heavy ions at GSI, in Proceedings of SRF'17 Conference, Lanzhou, China (2017), p. MOPB023.

[48] W. Barth et al., A superconducting CW-LINAC for heavy ion acceleration at GSI, EPJ Web Conf. 138, 01026 (2017). 